\title{
LA CONCEZIONE DEL TEMPO IN GUGLIELMO DI OCKHAM
}

Alessandro Ghisalberti*

SINTESE - A Física, desde Aristóteles, perguntouse sobre a noção de tempo. Guilherme de Ockham, ao tratar do assunto, critica a concepção desenvolvida por seus predecessores, fundamentada em uma leitura ontológica, enquanto ele opta por uma leitura baseada na empiria, o que o leva a considerar o tempo como um ente de razão. Com isso, abre caminho para submeter as categorias da temporalidade à análise lingüística.

PALAVRAS-CHAVE - Guilherme de Ockham. physics. Temporality. Ontology. Empeiria. LinguisFísica aristotélica. Temporalidade. Ontologia. tic analysis.

Empiria. Análise lingüística.

\section{La critica di Ockham alla concezione del tempo dei suoi predecessori}

Nel corso del sec. XIII, soprattutto ad opera di Tommaso d'Aquino, si è verificato il passaggio da un'ermeneutica del tempo legata ai referenti fisici e cosmologici, che appare essere quella preminente nel testo aristotelico, ad una dottrina della temporalità più marcatamente ontologica, che mette in stretta connessione il rapporto causale della durata o estensione della permanenza del movimento con la natura qualitativa specifica dell'essere che si intende misurare. La causa dell'eternità, come peculiare misura della durata di Dio, è data dall'essere perfettissimo, totalmente primo ed immobile; l'evo è la misura delle sostanze spirituali create, e ciò a causa del loro essere misurabili estrinsecamente, in rapporto al loro coesistere con un movimento regolare ed uniforme, ma anche a causa della loro invariabilità intrinseca, legata alla perfezione formale delle nature; causa del tempo, come misura degli enti generabili e comuttibili, è il loro essere intrinsecamente mutabile, la cui durata è segnata in modo strutturale e congenito dal moto (fa eccezione a ciò l'anima dell'uomo, equiparata alle sostanze angeliche).

* Università Cattolica del Sacro Cuore, Milano.

\begin{tabular}{|l|l|l|l|l|l|}
\hline VERITAS & Porto Alegre & v. 45 & n. 3 & Setembro 2000 & p. $381-392$ \\
\hline
\end{tabular}


La lettura ontologica è stata perciò veicolata dalla domanda circa la causa delle differenti misure della permanenza nello stato di movimento o di quiete, che determinano le differenze di durata in Dio, negli angeli e nelle sostanze ilemorfiche; la rilevanza metafisica della causalità proveniva soprattutto dalla tradizione neoplatonica ed aveva trovato nello pseudo-aristotelico Liber de causis il vertice della speculazione ontologico-aitiologica; a quest'opera l'Aquinate aveva dedicato un commento analitico proprio negli ultimi anni della sua vita.

Dalla prospettiva ontologica della durata prende le distanze Guglielmo di Ockham, il quale cala la portata della causalità nell'ambito dell'empinia, recedendo dall'ontologia della causa universale in riferimento ai generi e alle specie, la cui esistenza come universali viene ricondotta alla funzione significativa dei segni mentali, orali o scritti. La lettura ockhamiana si colloca in continuità con l'aristotelismo medievale posteriore a Tommaso d'Aquino, il quale si era riproposto un recupero del pensiero specifico dello Stagirita, in un'ottica di fedeltà alla lettera dei testi e contraria alla sovrapposizione di dottrine di matrice platonica, verificatasi soprattutto nei secoli V e VI, ad opera dei discepoli di Proclo e di Ammonio di Ermia (Simplicio, Asclepio, Giovanni Filopono). ${ }^{1}$

Questo processo di recupero del genuino aristotelismo si è imbattuto in diverse difficoltà, tra cui fu rilevante quella circa una possibile conciliabilità tra alcuni assunti della fisica aristotelica ed alcuni enunciati basilari della teologia cristiana; il nodo si aggrovigliò quando, nel marzo del 1277, il vescovo di Parigi Stefano Tempier emanò un Sillabo in cui venivano indicate 219 proposizioni come passibili di interpretazione errata, qualora fossero lette in modo conflittuale con dottrine connesse con il dogma cristiano. Nell'elenco di Denifle-Chatelain, la proposizione 49 recita: "Dio non può muovere il cielo di moto rettilineo". ${ }^{2}$ La tesi che ritiene impossibile per Dio imprimere un movimento rettilineo all'universo, se si parla non distinguendo tra possibilità assoluta e relativa, può creare conflitto con il dogma cristiano dell'onnipotenza divina; essa è un corollario della fisica aristotelica, secondo la quale, perché si possa produrre un moto rettilineo dell'universo nel suo insieme, ci sarebbe bisogno di un luogo contenente, capace di svolgere la funzione di luogo vuoto in relazione al termine del movimento: ma per Aristotele l'esistenza del vuoto è un assurdo.

La censura del Tempier sollecitò i pensatori latini che volevano preservare sia la dottrina aristotelica, sia la teologia cristiana, a cercare una spiegazione del movimento che permettesse di affermare la possibilità del moto anche quando non c'è un altro corpo (contenente) oltre al mobile. Pierre Duhem ${ }^{3}$ ha riscontrato come siano stati Duns Scoto ed i suoi seguaci i primi a proporre una soluzione a questo problema: il movimento locale non può essere limitato all'acquisizione dell'ubi da parte del mobile, ma comporta altresi l'acquisizione di una forma inerente al mobi-

1 Cfr. A. Ghisalberti, Percorsi significativi della Metafisica di Anistotele nel Medioevo, in "Rivista di Filosofia Neoscolastica" LXXXV (1993), p. 585-604.

Cfr. P. DUHEM, Le système du monde, VII, Parigi 1956, pp. 203-461. 
le, reale di una realtà puramente successiva e non permanente. Il moto locale deve cioè consistere in una forma realmente distinta sia dal mobile, sia dall'ubi, in grado di darsi anche quando l'ubi non c'è; tale forma venne denominata forma fluens (più tardi si è sovrapposto a questo nome quello di fluxus formae, pur non del tutto identico al primo).

Siffatta concezione del movimento coinvolse inevitabilmente la riflessione sul tempo, sotto la spinta di un'altra proposizione censurata dal Tempier, la n. 200: "L'evo e il tempo non esistono nella realtà, ma solo nella conoscenza". ${ }^{4}$ Andando alla ricerca della realtà posseduta dal tempo al di fuori della mente, i discepoli di Duns Scoto stabilirono che il tempo è forma fluens (o fluxus formae): Giovanni di Bassoles e Francesco di Meyronnes definirono il tempo fuori dall'anima come una realtà successiva analoga $\mathrm{al}$ movimento. ${ }^{5}$

Il riferimento a questi antecedenti storici serve a collocare la posizione di Ockham, il quale nelle diverse opere in cui tratta la dottrina del tempo, inizia sempre dalla critica alla concezione del tempo come entità distinta dalle res permanentes (il mobile ed il moto), critica che segue a quella di chi attribuisce al moto una realtà successiva, distinta da quella del corpo mobile che prima sta in un luogo e poi in un altro luogo, successivamente e senza quiete intermedia. ${ }^{6}$ Fra le argomentazioni contrarie all'attribuzione al tempo di una realtà successiva, di una qualità che si svilupperebbe nel fluire della forma verso il fine del movimento, la più consona alla metodologia ockhamiana è costituita dall'applicazione del principio di economia (il cosiddetto "rasoio di Ockham"), per cui: "frustra fit per plura quod potest fieri per pauciora", ossia si devono preferire le spiegazioni che ricorrono al minor numero di postulati; siccome per spiegare il moto e il tempo bastano le realtà permanenti, non si deve ipotizzare l'esistenza di altre realtà. La confutazione della possibilità di un'esistenza autonoma del tempo è svolta anche con molti argomenti puntuali; anzitutto non si può dire che sia l'ordine delle parti a costituire la realtà del tempo, perché ciò che non esiste, non può avere un ordine positivo: ora, le parti del tempo, nell'ipotesi che siano distinte dalle realtà permanenti, 0 sono passate o sono future, ossia non sono attualmente esistenti; di conseguenza non possono nemmeno essere disposte ordinatamente.

Ancora: non può costituire la realtà del tempo la continuità fornita dall'istante, perché se le parti del tempo sono un puro nulla, perché sono o passate o future, non possono avere nemmeno continuità alcuna fra loro, fornita da un qualche ente.

Se il tempo è un accidente, una successione fluente rilevata dall'anima, come prospetta la tesi degli scotisti, ci si chiede quale sia la sostanza di cui il tempo è accidente; tale sostanza può essere solo il mobile, ma è impossibile che il mobile

Denifle-Chatelain, Chartulanium, I, p. 554.

Cfr. P. DUHEM, Le système du monde, VII, pp. 312-316.

Cfr.: OCKHAM, Summula philosophiae naturalis, 1. IV, c. 2; Opera Philosophica, VI, St. Bonaventure 1984, pp. 347-349. Quaestiones in libros Physicorum Aristotelis, q. 37; Opera Philosophica, VI, pp. 493-495. Expositio in libros Physicorum Aristotelis, 1. IV, c. 18; Opera Philosophica, V, St. Bonaventure 1985, pp. 199-201.

OCKHAM, Quaestiones in libros Physicorum, q. 37; pp. 494-495. 
abbia un accidente avente le caratteristiche prospettate dalla tesi avversaria, perché non si vede dove si trova nella sua costituzione intrinseca tale accidente: o una parte del tempo è in una parte del mobile, e un'altra parte è in una parte diversa; oppure più parti del tempo sono in un'unica parte del mobile. Ma nessuna delle due ipotesi è sostenibile, "poiché non c'è motivo perché una parte si trovi in una determinata parte e l'altra in una parte diversa, né convince la disposizione inversa, come è evidente". ${ }^{8}$ Se poi tale accidente venisse inteso come accidente di un esteso indivisibile, e perciò venisse esso stesso raffigurato come esteso ed indivisibile, ne seguirebbe che tutto il tempo esisterebbe simultaneamente, perché tutte le parti del tempo sarebbero in tutte le parti del mobile; parti del tempo, e cioè il passato e il futuro, che peraltro sono un puro nulla, e perciò non possono positivamente coesistere con un ente reale, dal momento che il nulla non può né coesistere con ente alcuno, né formare una totalità o una unità. ${ }^{9}$

La stringenza logica delle argomentazioni di Ockham, fondata sull'impossibilità in fisica di attribuire consistenza reale a qualcosa di empiricamente non verificabile, lo porta all'affermazione centrale circa il tempo: "il tempo è un nome relativo o quanto meno connotativo"; "il tempo ha solo una definizione che esprime il significato nominale, come accade per i nomi, i verbi, gli avverbi, ecc.; [...] il nome tempo significa il moto nel caso retto e l'anima ed il suo atto nel caso obliquo". ${ }^{11}$

La valenza piena del tempo è svelata dalla rigorosa analisi linguistica, che lo sottrae ad ogni ipostatizzazione, ad ogni superficiale tendenza alla reificazione di un'entità concettuale: 'La proposizione 'il tempo esiste' non è da ammettere semplicemente, alla stregua delle proposizioni 'l'uomo esiste', 'la bianchezza esiste'; essa deve essere risolta in un'altra proposizione, poiché attraverso la proposizione 'il tempo esiste' non si deve intendere se non che qualcosa si muove, per cui l'anima può conoscere quanto un altro mobile si muove". ${ }^{12}$

L'analisi del tempo si colloca, per Ockham, tutta a livello del linguaggio scientifico: il tempo non possiede una realtà autonoma, ma è un temine che connota, associandole, due realtà oggettive, quella del mobile in movimento e quella del soggetto che misura il movimento secondo la successione di prima e dopo. La proposizione: "il tempo è", è un complexum, una articolazione di termini che ha valore sul piano della proposizione in cui i termini stanno in luogo delle entità naturali che essi significano. Per stabilire la portata scientifica di tali proposizioni si deve ricorrere alla virtus sermonis, alla esatta significazione dei termini, alla loro virtus o capacità semantica originaria, in rapporto alla quale i temini stessi sono stati istituiti. Così, nel nostro caso, il termine tempo non è stato istituito per significare delle realtà autonome, bensi per esprimere brachilogicamente questa proposizione: "aliquid movetur, unde potest anima cognoscere quantum aliquod aliud mobile movetur".

Ibidem, p. 494.

Cfr. ibidem, p. 495.

Ibidem.

Ibid., q. 47, pp. 524-525.

Ibid., q. 39; p. 500. Cfr. OCKHAM, Expositio in libros Physicorum, IV, 18; p. 199-201. 
E ora importante sapere se in questa prospettiva, si poteva affermare quanto postulava l'istanza avanzata dal Tempier, ossia che "tempus est ens", che il tempo è un ente. Ockham risponde affermativamente, ma con una spiegazione che va in direzione opposta a quella dei predecessori e dei contemporanei: egli precisa subito che il tempo è un ente nello stesso modo in cui è un ente il moto, e che le proposizioni: "tempus est", "motus est", pur godendo di una veritiera virtus sermonis, non vanno intese come le proposizioni: "homo est", "albedo est", le quali sono immediatamente risolvibili nei loro incomplexa, nelle realtà significate dai termini; le prime sono piuttosto il concentrato, per motivo di brevità, della proposizione più lunga sopra ricordata. In conclusione, equivalendo alla proposizione: "qualcosa si muove, per cui l'anima può conoscere quanto un altro mobile si muove", equivalendo cioè ad un enunciato o complexum, il tempo è sì un ente, ma è un ens rationis. Che cosa Ockham intenda per ens rationis lo stabiliamo sulla base della sua dottrina dei concetti universali, che sono enti (perché qualità) di ragione (perché qualità della mente), allocati nell'atto della intellezione.

Rispondendo all'obiezione relativa a come possa essere vera la proposizione "tempus est ens", stante che essa comporta l'ammissione dell'esistenza di realtà composte di non enti (il tempo si compone di passato e di futuro, ossia di enti che non sono, perciò di non enti), Ockham ribadisce che nulla può comporsi di parti non attualmente esistenti; la soluzione viene ancora una volta dalla vigile analisi linguistica, la quale avverte che, allorché si parla del tempo come risultante da enti che non sono, ossia dal passato e dal futuro, si vuole solo dare una definizione nominale del termine tempo, spiegarne il quid nominis. Perciò nella definizione "il tempo si compone di cose che non sono, ossia del passato e del futuro", il termine tempo non è preso nella supposizione personale, bensì secondo la supposizione semplice, quella di un concetto, la quale dà luogo alla definizione nominale. In questo senso è vero che la nozione di tempo implica che qualcosa è passato e qualcosa è futuro; ma non per questo le realtà extramentali cui fa riferimento si compongono di passato e futuro.

In sintesi, per Ockham il tempo è un ens rationis; come tale esso sta al posto, nel senso della supposizione personale, di un complexum, ossia della proposizione: "qualcosa si muove, per cui l'anima può conoscere quanto un altro mobile si muove"; le singole nozioni (incomplexa) in essa presenti hanno riferimenti precisi alle realtà extramentali, e consentono di dare la definizione reale di tempo, secondo il quid rei.

Nella proposizione che esprime la realtà del tempo balza subito agli occhi l'inclusione della medesima proposizione che, per Ockham, soggiace al termine moto: "aliquid movetur"; il primo problema da affrontare è perciò quello del rapporto tra le proposizioni indicate dai due termini, tempo e moto, per vedere se esse suppongano per le stesse realtà extramentali e dunque, secondo il quid rei, finiscano con l'esprimere la stessa cosa.

A questa domanda Ockham risponde senza esitazione in modo affermativo: il tempo infatti è il movimento grazie al quale l'anima conosce quanto dura un altro movimento; perciò il tempo non può supporre per una realtà distinta dalle realtà 
permanenti significate dal moto. ${ }^{13}$ Ciò non significa che i due termini in questione si risolvano nella medesima proposizione, e che perciò abbiano la stessa definizione nominale; il tempo significa di più, rispetto a quanto è significato dal moto, perché fa riferimento all'attività misurante dell'anima, e pertanto i due termini non sono interscambiabili fra loro, né sono sinonimi. I passi in cui Aristotele ed Averroè dicono che il tempo non è il moto, sono esattamente rivolti a spiegare come le due proposizioni in cui si risolvono non hanno la medesima definizione nominale, relativamente a ciò che significano i nomi tempo e moto, ${ }^{14}$ mentre la proposizione: "tempus est motus" è vera de virtute sermonis, badando cioè alle realtà extramentali significate dai due termini, le quali sono esattamente le stesse.

\section{Ockham sottopone le categorie della temporalità all'analisi logico-linguistica}

La ricostruzione puntuale della metodologia esegetica ha offerto un valido ausilio per comprendere le motivazioni che sottostanno alle soluzioni avanzate da Ockham in risposta ai diversi aspetti del problema; intenderei ora procedere ad una presentazione sintetica delle prese di posizione del Venerabilis Inceptor circa le più significative questioni specifiche sulla temporalità, dibattute nella tradizione dell'aristotelismo antico e medievale.

2.1 L'affermazione che il tempo è passio motus non va presa secondo la supposizione personale, la quale rinvia alle realtà extramentali, ma secondo la supposizione semplice o quella materiale, che fanno riferimento al termine mentale, orale o scritto; a livello di definizione nominale, i termini tempo e moto differiscono, perciò il tempo suppone per qualcosa in più del termine moto, e precisamente per l'anima numerante, la quale non è passio del movimento in senso formale. ${ }^{15}$

2.2 I moto particolarmente idoneo per essere usato dall'anima misurante è il movimento perfetto, uniforme e velocissimo, del primo mobile: la regolarità di questo movimento consente di misurare in modo uniforme la durata degli altri movimenti; la sua estrema velocità permette di misurare qualsiasi movimento, per quanto rapido. ${ }^{16}$

Va anche qui precisato, che tempo e moto del primo cielo coincidono de virtute sermonis, cioè secondo la realtà extramentale significata, ma non si tratta di sinonimi, per cui il termine "orbita" e il termine "tempo" non sono convertibili, e se ci fossero più moti nel cielo, non per questo ci sarebbero più tempi, dal momento che il concetto di tempo implica la misurazione, e quindi l'assunzione di un unico moto che funga da unità di misura per l'anima misurante.

13 OCKHAM, Quaestiones in libros Physicorum, q. 40; p. 503.

15 Ibid., q. 41; p. 506.

Cfr.: ibi, q. 44; pp. 516-517; OCKHAM, In II Sent., q. 10; Opera Theologica, V, St. Bonaventure 1981, p. 226.

${ }^{16}$

OCKHAM, Quaestiones in libros Physicorum, q. 42; pp. 508-510. 
2.3 E lecito avallare la distinzione tra tempo principale e tempi secondari, facendo riferimento per questi ultimi a movimenti inferiori rispetto a quello del cielo, i quali possono servire come unità di misura della durata dei movimenti particolari. Noi ci serviamo del moto dell'orologio, anch'esso uniforme e regolare, per misurare sia la durata del sole (quando è coperto dalle nubi), sia le nostre operazioni. Anche altri moti possono essere usati come tempi: sapendo quante miglia un cavallo percorre in un giomo, possiamo calcolare quanto tempo è trascorso sulla base dello spazio percorso dal cavallo; lo stesso vale per il moto, ben più regolare, dei pianeti. $^{17}$

2.4 La domanda se possa bastare un "motus imaginatus" per misurare il tempo consente ad Ockham di puntualizzare la consistenza del tempo nell'anima: basta un concetto dell'anima atto a misurare le cose fuori di essa, come è il concetto di numero, a garantire il rapporto tra la quantità immaginata e la quantità (di moto) della cosa extramentale. Nell'anima dunque il tempo possiede un'esistenza particolare, noetica, che consente alle parti del tempo di coesistere simultaneamente: "Concedo pertanto che il tempo nell'anima possiede le parti tutte insieme; ciò equivale a dire che l'atto di intendere con cui intendo una parte del moto e l'atto con cui intendo un'altra parte coesistono; ripugna tuttavia al tempo fuori dell'anima avere delle parti coesistenti". ${ }^{18}$ Non sfugge qui al lettore l'esplicita ripresa della difinizione agostiniana di tempo come "distensio animi", come attività della mente che accoglie in sé passato, presente, futuro, suggerita dall'istanza di attenzione fenomenologica che accomuna la ricostruzione del volto del tempo in Agostino e in Ockham.

2.5 Molto lucida è anche la soluzione ockhamiana della vexata quaestio scolastica se il tempo sia numero numerato o numerante: Ockham dice che il tempo ha caratteristiche tali per cui va detto sia numero numerato, sia numero numerante, perché, se si bada all'esatta accezione del termine, si possono mantenere entrambe le tesi.

Ci sono quattro modi di intendere il numero: a) il numero in quanto coincidente con la cosa numerata fuori dall'anima (dieci cani o dieci cavalli), e questo è il numero numerato; b) il numero col quale numeriamo, determinato in base alla misura di una cosa fuori dall'anima che ci è nota: è il caso dell'ulna (braccio), misura nota, con cui misuriamo la grandezza di altre cose. Tale numero non è inerente (ossia è distinto per sito e luogo) alla cosa numerata; c) il numero come atto dell'anima col quale misuriamo le singole cose numerate; d) il numero inteso come concetto, e come tale comune a molti e predicabile di molti: anch'esso viene detto numero col quale misuriamo le altre cose, poiché è una qualità della mente, è un atto uno e semplice, che può essere usato per misurare una molteplicità di realtà. Ora, osserva Ockham, la discussa esemplificazione di Aristotele col numero 
dieci cani e dieci cavalli può benissimo essere spiegata assumendo il termine in tutti e quattro i modi di intendere il "numero" sopra riferiti, ed in rapporto alle singole angolature di significato può risultare vera. La conclusione è chiara: Aristotele, allorché esemplifica il numero dal tempo paragonandolo al numero di dieci cani e dieci cavalli, si riferisce alla quarta accezione di numero, ossia al numero dieci (astratto), che è identico nelle due serie, benché nella realtà gli animali numerati siano diversi. Altrimenti detto, per Ockham il tempo è sia numero numerante, sia numero numerato, in base a diverse supposizioni significative del termine numero: $:^{19}$ è numero numerato, in quanto coincide con il moto diurno del cielo, realmente esistente fuori dall'anima; è numero numerante in quanto è il numero (esistente solo nell'anima), col quale l'anima "attraverso il moto diumo misura il prima e il poi negli altri moti". ${ }^{20}$

2.6 La realtà del tempo, in ultima analisi, sta più nel succedersi del movimento o nell'attività soggettiva dell'anima che misura? La complessità del tempo non consente ad Ockham di dare una risposta semplice ed univoca; egli preferisce perciò rispondere dicendo che il tempo possiede un'accezione propria e un'accezione impropria: nell'accezione propria non si può parlare del tempo come di una cosa composta da un ente nell'anima e un ente extra animam, perché nessuna entità in atto può risultare dalla composizione di una forma e di una materia intese in questo modo. Propriamente, il tempo non può essere caratterizzato da una duplice consistenza, oggettivabile in un ente dotato attualmente sia di un carattere mentale, sia di un carattere extramentale. In modo improprio, facendo riferimento quindi alla definizione nominale, descrittiva ed evocativa dei contenuti del tempo, si parla di inclusione nel tempo oltre che del movimento attuale, anche del movimento passato e di quello futuro, dotati solo di esistenza intenzionale, nella mente.

La forma del tempo, che è la numeratio attraverso l'anima, impedisce di parlare del tempo nella direzione dell'impossibile monstrum appena prospettato, ma porta a ragionare di un duplice essere del tempo, un essere attuale e un essere potenziale: "ciò che viene numerato può esistere senza l'anima, e perciò si dice che l'essere del tempo fuori dell'anima è in potenza, mentre il suo essere nell'anima è in atto; ovverossia, quando è numerato dall'anima, allora è numerato attualmente" ${ }^{21}$ Il tempo è costituito attualmente, e perciò nella sua piena formalità, dall'attività dell'anima misurante, mentre la sua materia è data dal movimento continuo. La stessa definizione del tempo come mensura motus appella alla sua forma, dove l'appellatio è il rinvio semantico proprio e peculiare del termine; è la "forma" semantica del tempo che appellat l'attività del misurare, come nota formale del tempo rispetto all'aliquid movetur, rispetto a ciò cui rinvia il termine moto.

19
Cfr. Ibid., q. 46; pp. 521-523.

Ibid., q. 47; p. 526.

Ibid., q. 50; p. 534; cfr. ibid., q. 53; p. 539. 
2.7 Una ammissione che Ockham fa sulla scia di Averroè merita di essere ricordata: si tratta della possibilità che esista un tempo discreto, possibilità ignorata da Aristotele, per il quale in tempo è sempre un continuo, data la sua relazione al movimento di traslazione continua del cielo. Affermando le possibilità del tempo discreto, non si vuole concedere che il tempo consti di due realtà distinte, di cui una continua e l'altra discreta, ma solo che "nella definizione del tempo si pone un nome che implica il continuo, cioè il moto, e si pone un altro nome che implica il discreto, cioè il numero"; "nella definizione del tempo si pongono due nomi, l'uno dei quali, il moto, significa che esiste qualcosa di continuo, e l'altro, il numero, significa che esiste qualcosa di discreto" ${ }^{22}$ La qualifica di discreto non è una nota che viene al tempo dalla condizione oggettiva extramentale, connessa con dei dati frazionati e divisi del mobile, ma semplicemente dal fatto che la discretio deriva al tempo dall'imprescindibile attività numerante dell'anima.

2.8 La rigorizzazione semantica dei termini guida Ockham anche nello svolgimento dei temi che a Tommaso avevano consentito di incentrare il tempo principalmente sulla nozione di durata, e di trasferime la trattazione sul piano dell'ontologia. Si tratta delle nozioni di istante e di eternità, con la connessa nozione di evo.

Circa l'istante, la tesi aristotelica asseriva che l'istante è il punto fisso che divide il passato dal futuro, punto presente e stabile, fissato in riferimento alla sfera celeste del primo mobile; la tesi di alcuni filosofi contemporanei ad Ockham, assertori dell'esistenza di realtà fluenti e successive, concepiva l'istante come una realtà indivisibile, distinta da qualsiasi cosa permanente, con la caratteristica di essere fluente, ossia di sparire in un batter d'occhio. Ockham respinge quest'ultima tesi, per l'impossibilità di contrassegnarne la natura esatta nell'ambito delle categorie o predicamenti, non potendo l'istante così inteso essere né sostanza né accidente, comportando entrambe le ipotesi delle conseguenze contraddittorie. La posizione aristotelica viene ricondotta ad una esegesi lontana dall'oggettività ipostatizzante l'istante, e guidata da un'analisi semantica di termini: l'avverbio nunc, come i participi instans o praesens, indicano principalmente il primo mobile e nessun'altra realtà prodotta ex novo. La rotazione del primo cielo fa sì che ogni parte della sfera mossa venga successivamente a collocarsi in posizione opposta alle singole parti della terra; questa opposizione delle singole parti del cielo alle singole parti della terra non comporta nulla di oggettivamente nuovo, non comporta cioè la generazione o la corruzione di nuove sostanze; essa significa solo che, per uno spettatore, il primo mobile è ora in un luogo, ora in un altro, ed in questo modo il primo mobile viene detto l'istante, il punto di riferimento discriminante il prima ed il poi, in riferimento al quale l'anima misura il moto. Di conseguenza dire che ora (nunc) un dato oggetto è bianco, significa affermare che esso ha tale colore mentre il primo mobile è in una posizione dove non era prima e dove non sarà in seguito.

Ibid., q. 53; pp. 539 e 540. 
In conclusione, si deve dire che instans è un termine connotativo, il quale designa primariamente il moto del cielo e secondariamente connota l'anima, che misura la quantità del movimento grazie al riferimento alla posizione del cielo, il quale, nel suo spostarsi di luogo, resta in sé identico e muta solo il situs (prima in un sito, poi in un altro). La virtus sermonis attesta che l'istante è un avverbio (nunc) che equivale ad un termine sincategorematico, e perciò non ha in sé significato definito, ma lo assume in rapporto al termine cui viene associato; come termine categorematico, l'instans è il primo cielo, esistente in un sito, ed in riferimento a questo sito non si verificano i contraddittori, perché esso consente di distinguere gli accadimenti secondo il prima ed il poi. Questo uso categorematico del termine risponde alle esigenze linguistiche avanzate dai filosofi, in primo luogo da Aristotele e da Averroè. ${ }^{23}$

La lettura di questi autori fatta dagli scolastici latini, in particolare quella di Tommaso d'Aquino, non ha tenuto conto delle istanze semantiche dei termini e della coerenza logica interna alla dottrina di Aristotele, che rifuggiva da ogni ipostatizzazione di entità mentali o linguistiche, ricorrente invece negli esegeti neoplatonici dei sec. II-VI d.C., e da cui occorre liberare il testo dello Stagirita: "Perciò, propriamente parlando, non si deve concedere che il medesimo istante permanga sempre come una realtà permanente in altri in modo continuo, né si deve concedere che si tratti di due istanti differenti come di cose diverse, di cui l'una sia prima e l'altra dopo". ${ }^{24}$ Nessuna realtà autonoma va concessa all'istante, né nella prospettiva di una realtà permanente, né in quella di un fluire successivo.

2.9 La dottrina comune agli scolastici circa l'etemità come misura della prima causa, è per Ockham l'occasione per verificare la capacità della sua dottrina della temporalità di sottrarsi ad ogni aggancio ontologico. In primo luogo, scrive, "mi domando circa l'eternità: o è altra cosa rispetto alla prima causa, oppure coincide con la prima causa stessa". ${ }^{25}$ La prima ipotesi dà luogo ad esiti aporetici: se l'eternità fosse qualcosa di distinto dalla prima causa, si dovrebbe di conseguenza dire che Dio viene misurato da qualcosa di altro da sé, il che è assurdo perché Dio è infinito e non è rapportabile ad alcuna misura diversa dalla propria essenza. Oppure si dovrebbe dire che questo qualcosa di distinto dalla prima causa è il nulla, con la conseguenza, parimenti assurda, che Dio sarebbe misurato dal nulla. Non resta allora che la seconda ipotesi, ossia che l'eternità coincide con la prima causa; contro questa ipotesi Ockham solleva obiezioni: non è possibile che la prima causa sia misurata dalla prima causa stessa, e per conseguenza non è possibile che sia misurata dall'eternità, "poiché nulla è misurato primariamente da se stesso, ma qualsiasi ente venga misurato da una cosa o è misurato semplicemente dalla cosa, oppure da un'altra parte di sé". Ora, Dio non ha parti, e perciò non è misurabile dall'eternità intesa come una sua parte; né può essere misurato simpli- 
citer dall'eternità nel caso che questa coincida con la prima causa, sia per la ragione già vista ("nihil mensuratur seipso"), sia perché quando una cosa è misurata da un'altra cosa, accade che dalla quantità conosciuta dell'una si certifica la quantità ignota dell'altra: è invece assurdo che dalla quantità nota di una cosa si certifichi la quantità ignota della stessa cosa, poiché è assurdo che un'identica realtà sia contemporaneamente più nota e meno nota.

L'obiettivo di Ockham è quello di escludere che la nozione di eternità includa la nozione di misura, partecipi cioè all'idea di un'attività che misuri l'esistenza della causa prima. A parere del Venerabilis Inceptor l'eternità è un reale attributo di Dio, poiché Dio né è misurato né misura in modo alcuno, pur essendo egli realmente eterno: "ma non è etemo per una etemità che sia la sua misura, come Dio è veramente intelligente, ma non è intelligente per una intellezione che sia la sua misu$\mathrm{ra}^{\text {"2 }}{ }^{26}$

Il piano della trascendenza divina impedisce un linguaggio di misure: sarebbe un concedere troppo alla prevaricante teologia affermativa, quasi un assoggettare in qualche modo l'essere di Dio ad una misura; Ockham mantiene per Dio la qualifica di eterno, perché questo termine esprime esattamente una natura sovranamente trascendente, e perciò del tutto immensa nel senso etimologico di non-metior, incommensurabile.

Al linguaggio umano è invero concesso di pensare Dio come coesistente con il tempo, ossia con la misura del movimento degli enti oggetto di esperienza; è perciò accettabile l'affermazione che "Dio è nel tempo", a patto che si intenda l'essere nel tempo nell'accezione molto larga di "coesistere col tempo", e non già in quella ristretta di "misura attraverso cui veniamo a sapere quanto si estende la durata di qualche cosa".

Questa risposta introduce l'interrogativo circa l'aevum, o l'eternità partecipata, assunto tradizionalmente come misura della durata degli angeli. Per Ockham parlare di etemità partecipata significa fare in qualche modo riferimento all'eternità di Dio; ma ciò è inaccettabile per gli angeli, poiché significa non afferrare l'abisso che separa l'essenza divina, che non può non durare, ossia deve restare identica a se stessa, dall'essenza degli angeli, i quali sono creati da Dio e quindi possono essere distrutti da Dio in qualsiasi momento. L'evo, come un medio tra l'eternità e il tempo, è una nozione spuria, che non esibisce la possibilità di realizzarsi in una natura. Per quanto concerne invece la misurabilità della durata degli angeli, è logicamente o linguisticamente corretto dire che essi sono misurati dal tempo, perché grazie al tempo si può conoscere almeno parte della loro durata: essi coesistono col tempo, per esempio, per cento o per mille anni. Si deve tuttavia essere avvertiti che il tempo non ci permette di dire quanto gli angeli o gli enti sempiterni durino complessivamente, dal momento che essi eccedono la temporalità, cioè perdureranno anche quando il tempo non sarà più. ${ }^{27}$

26

Ibidem, pp. 542-543. Cfr. OCKHAM, In II Sententiarum, q. 11; Opera Theologica, V, St. Bonaventure, 1981; pp. 232-252. Sulla questione dell'evo in rapporto alla dottrina globale del tempo cfr. A. GHISALBERTI, Guglielmo di Ockham, Milano $1996^{4}$, pp. 158-174. 
Come bilancio sintetico, si può dire che l'esigenza di verificazione empirica, connessa con la fisica aristotelica, ha portato Ockham ad assegnare un riferimento oggettivo al tempo (il moto del primo mobile), che, per ironia della sorte, sarà destinato a non risultare tale con la rivoluzione scientifica copernicana e galileiana. Resta allora l'istanza metodologica connessa con la virtus sermonis, formidabile esempio dello sforzo di dare coerenza ad una dottrina fisicistica del tempo, senza cadere nell'incoerenza dell'ipostatizzazione di entità non empiricamente suffragabili. Una raffinata logica ha regolato l'incontro tra il principio di verificazione empirica ed il principio di verificazione linguistica, con la conseguenza che Ockham ha contribuito ad affinare il linguaggio sull'eternità di Dio e sulla durata delle sostanze sempiterne, oltre che a trasferire sul piano di quel particolare tipo di vissuto umano che è il linguaggio, sul piano della consuetudo loquentium, gran parte della dimensione soggettivo-antropologica inclusa nella riflessione agostiniana e neoplatonica sul tempo.

\section{Bibliografia}

AVERROEs In octo libros Physicorum, in Aristotelis Opera cum Averrois Commentariis, Venezia 1562. DenIFle H., Chatrl.AIN A., Chartulanium Universitatis Parisiensis, Parigi 1899.

DUHEM P., Le système du monde, VII, Parigi 1956.

GHISALBERTI A., Guglielmo di Ockham, Milano $1996^{4}$.

GHISALBERTI A., La nozione di tempo in San Tommaso d'Aquino, in "Rivista di filosofia neoscolastica", LIX (1967), pp. 343-371.

GhisalberTi A., Percorsi significativi della Metafisica di Aristotele nel Medioevo, in "Rivista di Filosofia Neoscolastica" LXXXV (1993), pp. 585-604.

OCKHAM, Expositio in libros Physicorum Aristotelis; Opera Philosophica, V, St. Bonaventure 1985.

OCKHAM, Quaestiones in librum secundum Sententiarum - Reportatio; Opera Theologica, V, St. Bonaventure, 1981.

OCKHAM, Quaestiones in libros Physicorum Aristotelis; Opera Philosophica, VI, St. Bonaventure 1984.

OCKHAM, Summula philosophiae naturalis; Opera Philosophica, VI, St. Bonaventure 1984.

TOMmaso D'AQuino, Commentaria in octo libros Physicorum Aristotelis, ed. Leonina, Roma $1882 .$. 